\title{
Study of Rotating Seat that Improves Vision of the Interior of an Automobile
}

\author{
Shoichiro TAKEHARA*, Yoshihiro SUDA** and Daisuke YAMAGUCHI** \\ *Sophia University, Department of Engineering and Applied Sciences, Faculty of Science and Technology \\ **The University of Tokyo, Institute of Industrial Science
}

Received August 1, 2013, Accepted February 24, 2014

\begin{abstract}
Recently, the demand for improvement of passenger comfort in automobiles has increased. An automobile does not have a large amount of volume to physical space, and space planning of the seat arrangement greatly influences passenger comfort. The seat arrangement changes the visual environment of the passenger. Previously, the authors proposed a technique to evaluate comfort through the seat arrangement by using the passenger's space and vision. In this evaluation technique, vision is recognized as volume. A useful seat arrangement with a rotated seat utilizes the effect of vision for improving passenger comfort. In this paper, a sensory evaluation and a biomedical measurement are performed. Therefore, the effectiveness of a seat arrangement with rotated seats is shown to improve passenger comfort.
\end{abstract}

KEYWORDS: automobile, comfort, vision, vehicle interior, seat arrangement, human engineering

\section{Introduction}

Recently, the demand for improvement of comfort in automobiles and railway vehicles has increased. Especially, automobiles having many seats, such as minivans, have become common and the vehicle interior has been accepted as a living room. However, automobiles have the feature of a large amount of volume that passenger occupies inside the vehicle. So, the space design of the vehicle interior has a big effect on comfort. Because comfort directly affects human emotions, it must be considered in automobile development. Therefore, quantitative evaluation of the comfort of the vehicle interior is very important. In the past, comfort has been evaluated based on vibration, motion or each factor as noise ${ }^{12)}$ but it has been difficult to quantify comfort by coordinating the effects of these factor. In this research, an experiment is performed to select and evaluate the factor of comfort in the vehicle interior. Then, the importance of vision is shown. The authors previously suggested a comfort evaluation for a seat arrangement that considers the sense of touch (physical volume), vision and psychology (visual volume) and showed the validity of this equation ${ }^{3}$. In this paper, the authors suggest a new seat arrangement for a minivan by applying the effect of vision to the abovementioned estimation equation of comfort. The seats in the second row rotate inward or outward of the minivan in this seating arrangement. To evaluate this arrangement, both a subjective estimation and a biomedical measurement in a driving simulator are performed. From the results of these experiments, the influence of the visual effect in the vehicle interior is discussed.

\section{Study of vision in the automobile}

In this section, the estimation equation of comfort in the vehicle interior, on which the proposal of rotated seats is based, is explained and application of the estimation equation is introduced.

\subsection{Evaluation factor of comfort in vehicle interior}

Here, two preliminary experiments are introduced. These preliminary experiments are performed to select the physical volume and the visual volume as variables used for the estimation equation of comfort.

As a fundamental study, these experiments extract the evaluation factor of comfort for a subject riding in an automobile in order to understand how the passenger environment influences comfort.

First, the evaluation factor of comfort in the stopped condition is extracted. In this experiment, four types of automobiles are selected from a sedan and a minivan, and the evaluation factor of six subjects is extracted by the evaluation grid method ${ }^{4}$. From the results, it is found that the evaluation factor related to width is selected under all conditions and the feeling of width significantly influences the comfort of the vehicle interior.

Next, a driving experiment is conducted to examine the importance of the resulting evaluation factor. In the experiment, eight subjects ride 10 min on a test course 
Table 1 Evaluation questions

\begin{tabular}{|l|l|l|l|}
\hline Question & example & Question & example \\
\hline \hline Spaciousness & Wide, narrow & Easiness for getting into car & Easy, diffecult to get on and off \\
\hline Stately & Stately, undignified & Cleanliness & Clean, dirty \\
\hline Atmosphere & Calm, rough & Quietness & Quiet, noisy \\
\hline Open & Open, close & Tightness & Tight, easy \\
\hline Safety & Safe, not safe & Mobility & Easiness, hardness to move in \\
\hline Individuality & Original, ordinary & Smell & Pleasant, bad smell \\
\hline Next person & Bothering, not botering & Tiredness & Tired, not tired \\
\hline Luxurious & High rank, low-end & Temperature & Warm, cool \\
\hline Brightness & Bright, dark & Stability on the seat & High stabilize, low stabilize \\
\hline Interest factor & Interest, not interest & Right and left side & Comfortable, uncomfortable \\
\hline Vibration & Shaky, not shaky & The front side & Comfortable, uncomfortable \\
\hline Manipulability & Good, bad manipulability & Seat back & Comfortable, uncomfortable \\
\hline Touch & Soft, stiff & Feet space & Comfortable, uncomfortable \\
\hline Material textures & Good, bad quality & Overhead clearance & Comfortable, uncomfortable \\
\hline
\end{tabular}

\begin{tabular}{|l|c|ccccc|}
\hline Evaluation & $\begin{array}{c}\text { Concrete } \\
\text { example }\end{array}$ & $\begin{array}{c}\text { not } \\
\text { important } \\
\text { at all }\end{array}$ & $\begin{array}{c}\text { not too } \\
\text { important }\end{array}$ & $\begin{array}{c}\text { somewhat } \\
\text { important }\end{array}$ & $\begin{array}{c}\text { considerably } \\
\text { important }\end{array}$ & $\begin{array}{c}\text { very } \\
\text { important }\end{array}$ \\
\hline \hline Spaciousness & wide or narrow & $\square$ & $\square$ & $\square$ & $\square$ & $\square$ \\
\hline
\end{tabular}

Figure 1 Example of the questionnaire

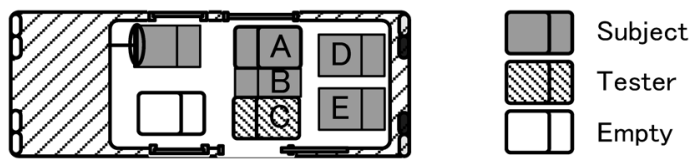

Figure 2 Seat arrangement of experimental car

that simulates roads in an urban area, in the country, and on a freeway. After the riding experiment is finished, the subjects fill out a questionnaire about the relationship between comfort and each evaluation factor. In this questionnaire, all automobiles are generally grouped together, and so a specific vehicle type or a specific seat in the automobile is not considered. The questions include the following: "Suppose you wanted to make a comfortable car environment. How important is each of the following items?" An example of the questionnaire is shown in Figure 1. The importance of the evaluation factors in the questionnaire is shown in Table 1. The question in Figure 1 was performed for each item in this table. The examples in this table are concrete examples of each item. Three types of automobiles having different automobile dimensions (interior length: $2500 \mathrm{~mm}, 2700 \mathrm{~mm}, 3000 \mathrm{~mm}$ ) are used to change the size of the vehicle interior. All automobiles have six passengers and a driver. Four subjects (seats A, B, D, $\mathrm{E}$ ), and the tester (seat C) sit as shown in Figure 2. The questionnaire results are shown in Figure 3. From these results, it is found that the factors related to the neighboring person and the factors related to space, such as foot space, are important. Other than the whole space around the seat, factors such as the right and left or front side around the passenger, the feeling of tightness, spaciousness, and so on, are related to vision. Therefore, these factors are found to be comparatively important.

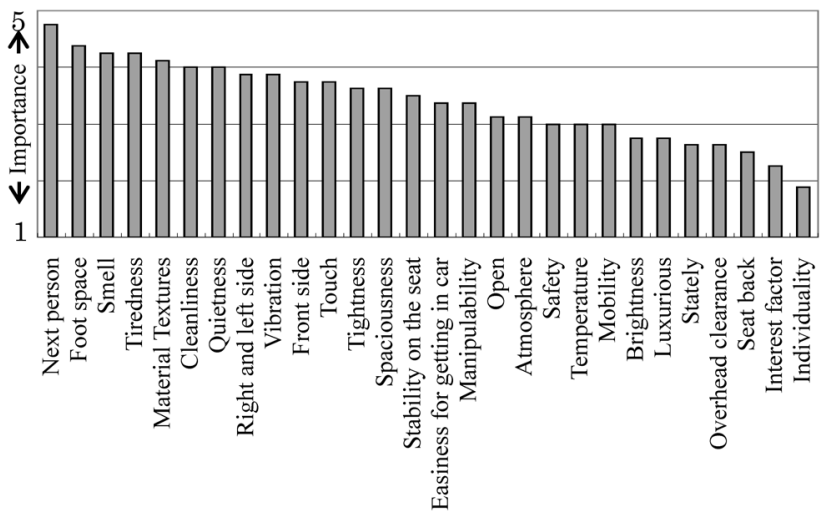

Figure 3 Importance of each evaluation factor

\subsection{Estimation equation of comfort in vehicle inte- rior}

In this research, the estimation equation of comfort in the vehicle interior is suggested. The equation is validated by comparison with experimental results using an actual automobile ${ }^{3)}$. In this section, the application of this equation is introduced.

The experimental results mentioned in Section 2.1 show the importance of vision for comfort in the vehicle interior, and so this equation is created by considering the effect of vision. The equation has two parameters of physical volume and visual volume. Physical volume is defined as the volume of space that the passenger can touch. Physical volume means the volume of actual space around the seat and it is generally measured in a traditional automobile evaluation as the space around the passenger's head and feet, and the right and left clearance. On the other hand, visual volume is defined as the volume of space seen by the passenger. Not only is visual volume an important physical quantity in this research, but also it is the original contribution of this research. The influence of hindering the view of a passenger by an obstacle can be shown quantitatively. This physical quantity is the volume of sectorial pillar space, shown in Figure 4.

Next, the reduction of comfort caused by neighbors and obstacles is explained. Generally, these factors are assumed to reduce comfort. In our equation, this effect is considered by subtracting the volume shown as the shaded area in Figure 4 from the physical and visual volumes. For example, the effect of a neighbor is considered by subtracting the shaded area that is the common area with the neighbor, because physical contact between the subject and the neighbor occurs and the effect of obstacles including the neighbor is considered by subtracting the volume of the obstacle and the visual volume blocked by the obstacle. Using sectorial volume, this equation is able to consider the effect of an obstacle close to the passenger that makes the passenger feel uncomfortable. Both of the physical and visual 


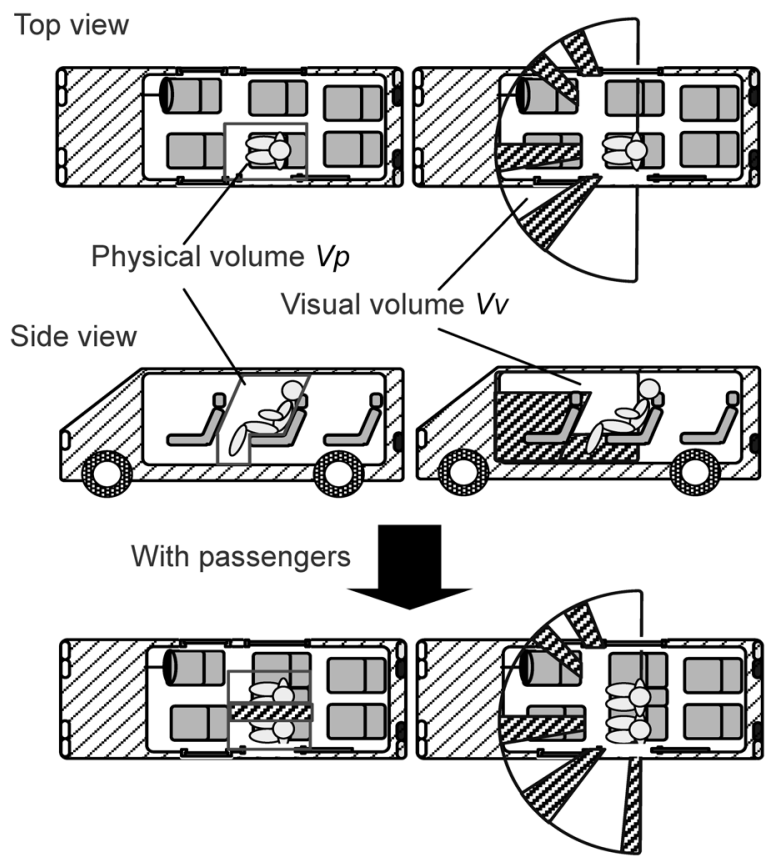

Figure 4 Concept of physical volume Vp (Left) and visual volume Vv (Right)

volumes are able to be calculated as a value from the drawing of an automobile in development. The estimation equation is as follows:

$$
\begin{aligned}
& C s(t)=\left(-A+A^{\prime} e^{-\frac{t}{T}}\right) V p+\left(B-B^{\prime} e^{-\frac{t}{T}}\right) V v-C+C^{\prime} e^{-\frac{t}{T}} \\
& A=1.56, A^{\prime}=1.66, B=2.56, B^{\prime}=0.66, C=1.89, C^{\prime}=0.68
\end{aligned}
$$

In this equation, $V p$ is physical volume, $V v$ is visual volume, and $C s(t)$ is the score of comfort at time $t$. Substituting the values of $V p$ and $V v$ evaluated by using the drawing, the comfort score can be estimated. $C s(t)$ is a seven-level evaluation. In this equation, $A, B, A^{\prime}$, and $B^{\prime}$ are coefficients, and $C$ and $C^{\prime}$ are constant numbers. Each coefficient and each constant number are calculated from the questionnaire score of experiments using multiple regression analysis. $T$ is a time constant. Here, $T$ is 2 from the experimental results. These values are evaluated by an identification experiment, which is an experiment using the minivan with 12 subjects. Further details of the derivation of this equation are described in reference ${ }^{3)}$.

\subsection{Utility seat arrangement using visual volume}

By using the abovementioned estimation equation, the improvement of comfort is discussed by giving an angle of rotation to the seats to increase the visual volume practically. In the focus on vision, interaction of the legs of the passengers is avoided. Therefore, seats $A$ and D are shifted $15 \mathrm{~cm}$ forward from seats $C$ and D. All seats are rotated equally, as shown in Figure 5. The relationship between the angle of rotation and comfort

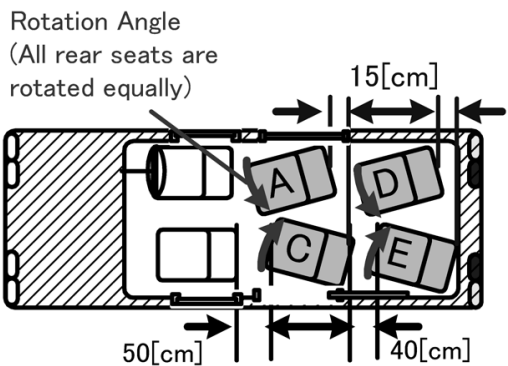

Figure 5 Rotation of seats
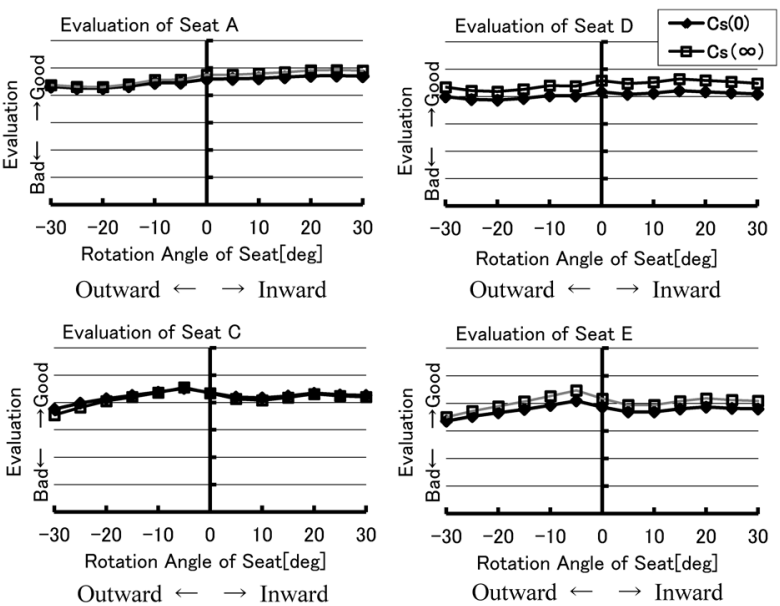

Figure 6 Score of comfort vs. angle of rotation

for all seats is shown in Figure 6. In this figure, $C s(0)$ is the comfort when passengers just ride in the vehicle and $C s(\infty)$ is the comfort when the time is infinite. The change of comfort, which is found for each angle, is caused by the change of the effects of the head rest of the front seat and the pillar on the vision. This means that visual volume can be changed by only rotating the seats.

\section{Visual effect by rotating seats}

In this section, the effect of vision by using rotated seats is discussed. This seat arrangement can also control the action of the human, who is affected by visual information.

As suggested in the preceding section, comfort is expected to be improved by making the visual volume bigger by using rotated seats. The most effective seat arrangement using rotated seats to improve comfort is investigated in a driving simulator ${ }^{5}$.

\subsection{Experimental condition in driving simulator}

To evaluate the effect of the proposed seat arrangement, a driving simulator is used. The advantage of a driving simulator is its ability to simulate the same situation many times. In this examination, the cut-body of an actual minivan is put on the driving simulator. A 
photo of the driving simulator is shown in Figure 7. The driving simulator is composed of a motion actuator of six axes and a full screen of 360 degrees.

The number of participants and the experimental condition are as follows;

(1) Subjects: 24 subjects (17 males and 7 females)

(2) Time of riding: $6 \mathrm{~min}$

(3) Riding speed: $60 \mathrm{~km} / \mathrm{h}$

(4) Angle of seat: 6 patterns (front, inward 5, 10 and 15 degrees, outward 5 and 10 degrees)

(5) Course scenario: Tokyo Expressway (Shinjuku Line) The ages of the subjects are 20 to $60 \mathrm{~s}$ and all have ridden in automobiles. Half of them ride automobiles only several times per year and others ride automobiles several times per week. In addition, conversation between the subjects is permitted. To simulate an actual ride, a driver sits behind the wheel.

The experimental procedure is as follows:

(1) Subjects sit down on one of the seats A, C, D and E at 0 degree.

(2) Subjects evaluate the questionnaire in the stopped condition.

(3) Subjects evaluate the questionnaire after the run.

(4) Subjects sit in another seat, and then (2)-(3) are repeated.

(5) The seat angle is changed, then (1)-(4) are repeated. The questions relate to comfort, communication, view, person in side seat, person in front seat, gap, motion sickness, and disgust. The evaluation is on a scale of $1-7$, and 7 is perfection. In addition, all subjects provided informed consent.

For the biomedical measurement, the change of viewpoint is measured by using a glass type recorder that produces a moving image of the viewpoint. In this measurement, the subjects sit in the second seat on the right-hand side. This experiment is different from the sensory evaluation experiment.

\subsection{Result of experiment using driving simulator} 3.2.1 Effect of change of vision by rotating seat

First, the result of the rotated seat is made into an

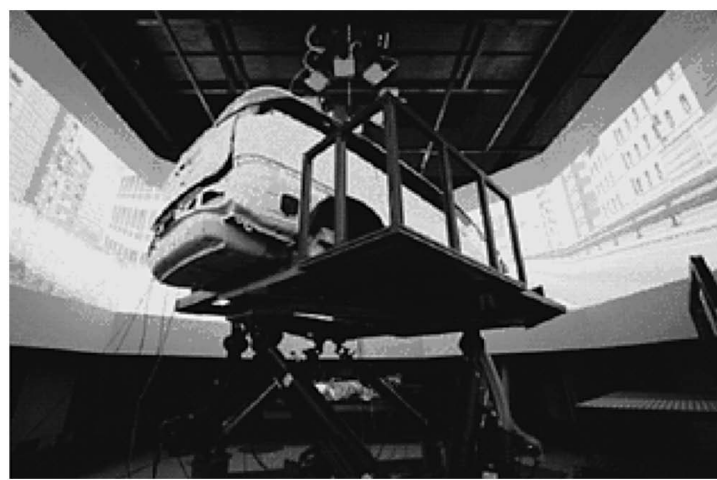

Figure 7 Driving simulator example, and its influence on each item is shown. This result shown in Figure 8 is the average evaluation of all seats for one subject. The horizontal axis is the angle of rotation of the seat and the vertical axis is the evaluation score. In this figure, the rotation angle inward is a positive value and the rotation angle outward is a negative value. The score is as described in Section 3.1. From this result it is found that comfort after starting to ride is improved when the seat is rotated inward. It turns out that at least only 10 degrees of rotation has effect on the score of comfort. Moreover, the evaluation of communication and view are improved when the seat is rotated inward. Additionally, the score of the neighbor is high when the seat is rotated inward. It is considered that seats rotated inward make it easier for this subject to talk with their neighbor because the neighbors are in the view of this subject. These results agreed with the concept of this seat arrangement. There is almost no change in motion sickness and disgust.

Next, the tendency of all subjects is investigated. A hierarchical cluster analysis is performed. This analysis uses the sum of the absolute value of the variation of the score of comfort after starting to ride, and the average score of the rotation inward and the rotation outward are used. From this result, four groups of subjects are formed: (a) prefer inward (the subjects in this group feel more comfortable after riding when the seat is rotated inward compared with an angle of 0 degree), (b) prefer outward (the subjects in this group feel more comfortable after riding when the seat is rotated outward compared with an angle of 0 degree), (c) prefer both inward and outward (the subjects in this group prefer comfort after riding better or the same when the seat is rotated inward or outward compared with 0 degree), and (d) prefer front (the subjects in this group feel more uncomfortable after riding when the seat is

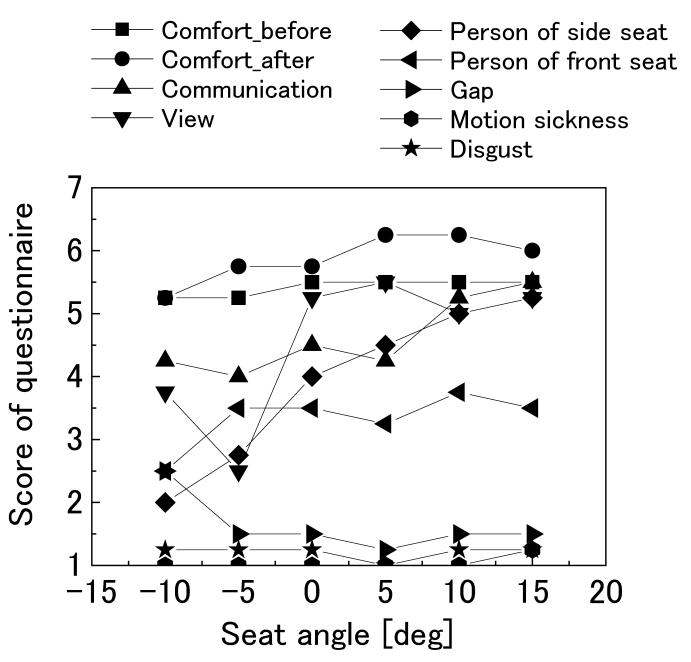

Figure 8 Experimental result of subject $A$ 
rotated inward or outward compared with an angle of 0 degree). For example, the breakdown about the right seat in the second row is shown as follows: six subjects are in group (a), eight subjects are in group (b), four subjects are in group (c), and six subjects are in group (d).

The average score of the experimental results of
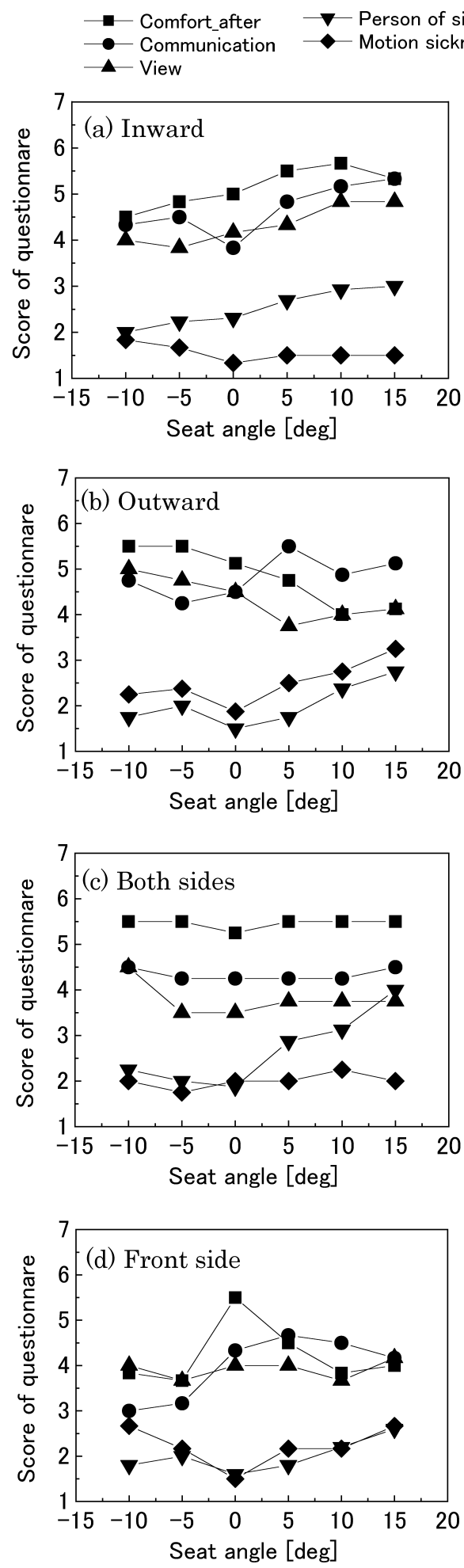

Figure 9 Experimental results of four patterns each group of subjects about the right seat in the second row is shown in Figure 9. In this figure, characteristic factor shown in Figure 8 is chosen. From these results, subjects in all groups pay attention to their neighbor when the seat is rotated inward. The scores of the comfort, the view and the communication are improved in inward for the group who prefers inward rotation. The reason why the score of the view is improved for this group is thought to be that there is space between the driver seat and front passenger seat and it is in front of the subject. For this group, the score of motion sickness moves up in outward. On the contrary, the score of the comfort and view are improved for the subjects in the group who prefer outward rotation. For this group the score of motion sickness moves up in inward. For the subjects in the group who prefer both sides, the effect of improvement of comfort by seat arrangement is smaller than that in the other groups. The score of motion sickness have almost no change. The scores of the comfort are not improved in inward and outward for the group who prefers front side. In all groups, motion sickness decreases comfort. Especially, subjects who prefer front seats feel motion sickness largely.

It means that the factor of motion sickness has greater influence on comfort than that of communication or view. As mentioned above, rotating seat can improve comfort for the half of subjects. On the contrary, this effect is reduced significantly by the influence of motion sickness.

\subsubsection{Influence of the view outside an automobile}

To investigate the difference of evaluations between left-hand seats and right-hand seats, the preferred rotation directions of right-hand and left-hand seats are classified by using the results of the questionnaires of all subjects. The results of the classification are shown in Table 2. The results show that approximately half of the subjects have a different preferred rotation direction in the right-hand seat or the left-hand seat. Especially, half of the subjects who prefer outward in the right-hand seat have a different preference for the lefthand seat. The subjects who have this tendency marked a higher score for the view of the outward direction in the right-hand seat than that in the left-hand seat. This is considered to be caused by the difference of right and left views. The subjects' vision is cut off by a wall and tunnel from the car window in the left-hand seat in the experimental road, as shown in Figure 10. For this reason, it is revealed that subjects who prefer an outward direction think a great deal of the view for comfort and this phenomenon shows the importance of design of the visual environment. 
Table 2 Change of one's favorite

\begin{tabular}{|c|c|c|c|c|c|}
\hline \multirow{2}{*}{ Subject } & \multicolumn{2}{|c|}{ 2nd Seat } & \multirow{2}{*}{ Subject } & \multicolumn{2}{|c|}{ 2nd Seat } \\
\hline & Left-hand & Right-hand & & Left-hand & Right-hand \\
\hline $\mathrm{a}$ & B & B & $\mathrm{m}$ & $\mathbf{O}$ & $\mathbf{O}$ \\
\hline $\mathrm{b}$ & $\mathbf{F}$ & B & $\mathrm{n}$ & $\mathbf{F}$ & $\mathbf{O}$ \\
\hline $\mathrm{c}$ & B & B & 0 & I & $\mathbf{O}$ \\
\hline $\mathrm{d}$ & B & B & $p$ & B & $\mathbf{O}$ \\
\hline $\mathrm{e}$ & $\mathbf{F}$ & I & $q$ & $\mathbf{F}$ & $\mathbf{O}$ \\
\hline $\mathrm{f}$ & I & I & $\mathrm{r}$ & $\mathbf{F}$ & $\mathbf{O}$ \\
\hline $\mathrm{g}$ & I & I & $\mathrm{s}$ & $\mathbf{O}$ & $\mathbf{F}$ \\
\hline $\mathrm{h}$ & $\mathbf{F}$ & I & $\mathrm{t}$ & I & $\mathbf{F}$ \\
\hline $\mathrm{i}$ & I & $\mathbf{I}$ & $\mathrm{u}$ & $\mathbf{F}$ & $\mathbf{F}$ \\
\hline $\mathrm{j}$ & I & I & $\mathrm{V}$ & $\mathbf{F}$ & $\mathbf{F}$ \\
\hline $\mathrm{k}$ & $\mathrm{O}$ & $\mathbf{O}$ & $\mathrm{w}$ & $\mathbf{F}$ & $\mathbf{F}$ \\
\hline 1 & $\mathbf{O}$ & $\mathbf{O}$ & $\mathrm{x}$ & $\mathbf{F}$ & $\mathbf{F}$ \\
\hline
\end{tabular}

I: Inward direction is comfortable

$\mathrm{O}$ : Outward direction is comfortable

B: Both inward and outward directions are comfortable

F: Frontal direction is comfortable

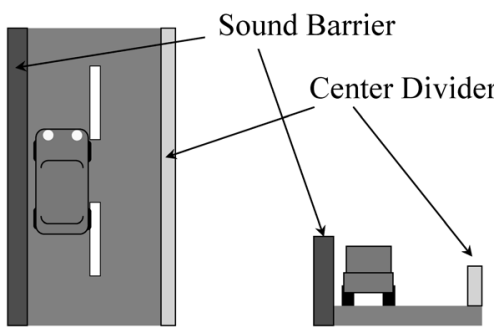

Figure 10 Reason of change

\subsubsection{Change of viewpoint}

From the standpoint of biomedical measurement, the influence of the rotated seat is investigated. The change of viewpoint is measured by a recorder of the glass type, which produces a moving image of the viewpoint. The image in the vehicle interior for seat $A$ is shown in Figure 11. In this figure, this image is divided into three categories: (a) left window, (b) front window and vehicle interior, and (c) right window. The time when the viewpoint stayed on each area is measured. The ratio of this time in total riding time is shown in Figure 12. From this result, subjects see outside the window for approximately $30 \%$ of the total time when the seat is rotated outward. On the other hand, subjects see the vehicle interior when the seat is rotated inward. This ratio increases by adding the angle of rotation. From this result of biomedical measurement, it is shown quantitatively that subjects tend to see the outside view naturally when the seat is rotated outward and they tend to see the vehicle interior when the seat is rotated inward.

\section{Summary}

In this paper, to improve the comfort of automobiles, the vision in a vehicle interior is investigated. The useful seat arrangement with rotated seats utilizes the effect of vision. From the results of sensory evaluation using a driving simulator, it is found that by changing

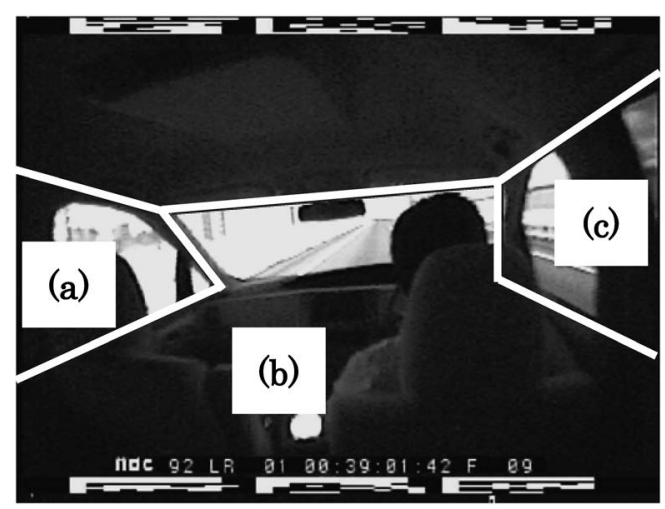

Figure 11 Area of sight

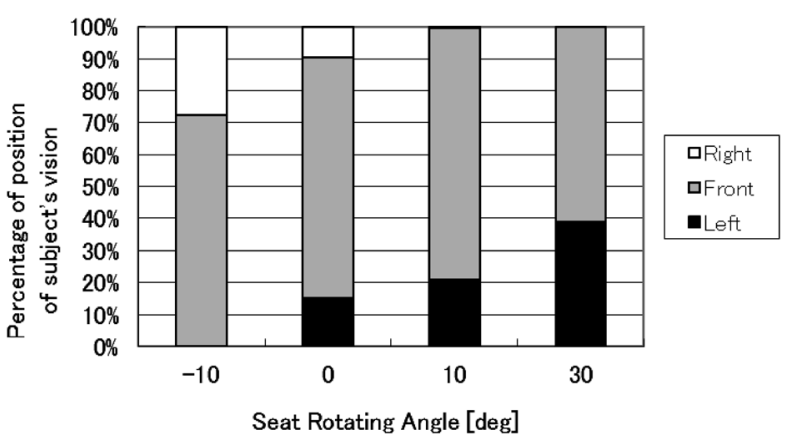

Figure 12 Proportion of seen area

the visual environment, the passengers are able to see outside the window more easily and to talk more easily. However, these effects apply not to all passengers. It is found that preference exists in each passenger. In this experiment, it turned out that the rotating seat has improvement effect of comfort for $60 \%$ of subjects. The improvement of comfort has an effect for at least 10 degrees of rotation. Moreover, the change of viewpoint is confirmed from the biomedical measurements. The tendency of this change corresponds to the results of the sensory evaluation. In an environment like the interior of automobile, the influence of motion sickness must be considered. Because the proposed seat arrangement is rarely different from usual seat arrangement, the result of this research suggests the possibility of engineering application of rotating seat.

\section{References}

(1) Suzuki, H.: Riding comfort and design of railway vehicles, J. Jpn. Soc. Design Eng., 34(6), pp. 190-195 (1990).

(2) Suzuki, H.: Human scientific approach to railway transport research: Recent topics and potential developments, RTRI report, Vol. 22, No. 7, pp. 1-4 (1998).

(3) Takehara, S., Kaneyasu, T. and Suda, Y.: Research 
on comfort evaluation in automobiles, Trans. Soc. Automotive Eng. Jpn., 39(1), pp. 139-144 (2008).

(4) Sanui, J. and Inui, M.: Phenomenological approach to the evaluation of places, J. Architect. Plan., 367, pp. 15-21 (1986).

(5) Suda, Y., Takahashi, Y. and Onuki, M.: The universal driving simulator for human, vehicle and traffic research, J. Soc. Automotive Eng. Jpn., Jpn.
Soc. Mech. Eng., 59(7), pp. 83-88 (2005).

Part of this work is published on the Transactions of Society of Automotive Engineering of Japan, Vol. 39, No. 1, pp. 139-144, (2008) and the Transactions of Society of Automotive Engineers of Japan, Vol. 41, No. 6, pp. 14391444 (2010). 